\title{
La técnica de biopsia de ganglio centinela prolonga la sobrevida libre de enfermedad en pacientes con melanoma
}

\author{
Sentinel-node biopsy prolongs disease-free survival in melanoma patients
}

Morton y col. NEJM 2014;370(7):599-609.

\section{Objetivos}

Evaluar si la incorporación de la biopsia de ganglio centinela mejora la sobrevida en pacientes tratados por melanoma.

\section{Diseño, lugar y pacientes}

Ensayo clínico aleatorizado multicéntrico en fase 3. Incluyó para el análisis a 1.661 pacientes con melanoma invasivo de espesor intermedio y grueso $(1,20$ a 3,50 y $>3,50 \mathrm{~mm}$, respectivamente [Clark III y Breslow $>1 \mathrm{~mm}$; o Clark IV/V con cualquier Breslow]). Estos fueron reclutados entre 1994 a 2002 y seguidos durante diez años tras la aleatorización.

\section{Intervención y medición de resultados}

En ambos grupos se realizó resección local amplia y observación de los ganglios regionales, con linfadenectomía en caso de metástasis. En el grupo intervención se realizó además, al momento de la resección amplia, identificación y biopsia del ganglio centinela, con linfadenectomía regional inmediata en caso de positividad (presencia de células tumorales en el ganglio biopsiado). La observación de la recidiva se realizó mediante examen clínico, laboratorio y radiografía de tórax cada tres meses durante los primeros dos años, cada cuatro meses durante el tercer año, cada seis meses durante el cuarto y quinto año, y anualmente hasta el décimo año de seguimiento. La aleatorización se realizó con una relación 60:40, y el análisis por protocolo. El resultado principal fue la sobrevida específica por melanoma. Dentro de los resultados secundarios se consideró también, sobrevida libre de enfermedad.

\section{Resultados}

La distribución de factores pronósticos entre los grupos estuvo balanceada excepto por la edad de los participantes que presentaban melanoma de espesor grueso. Los resultados principales y secundarios se detallan en la tabla 1.

Tabla 1. Sobrevida específica y libre de enfermedad por melanoma a diez años.

\begin{tabular}{l|c|c|c}
\multicolumn{1}{c|}{ Resultado } & $\begin{array}{c}\text { Grupo intervención } \\
\text { Media } \pm \mathrm{EE}\end{array}$ & $\begin{array}{c}\text { Grupo observación } \\
\text { Media } \pm \mathrm{EE}\end{array}$ & Media $\pm \mathrm{EE}$ \\
\hline Sobrevida específica & & & $78,3 \pm 2,0$ \\
\hline Melanoma de espesor intermedio & $81,4 \pm 1,5$ & $64,4 \pm 4,6$ & $0,84(0,64 \mathrm{a} 1,09)$ \\
\hline Melanoma de espesor grues0 & $58,9 \pm 4,1$ & & $1,12(0,76 \mathrm{a} 1,67)$ \\
\hline Sobrevida libre de enfermedad & & $64,7 \pm 2,3$ & $0,76(0,62 \mathrm{a} 0,94)$ \\
\hline Melanoma de espesor intermedio & $71,3 \pm 1,8$ & $40,5 \pm 4,7$ & $0,70(0,50 \mathrm{a} 0,96)$ \\
\hline Melanoma de espesor grues0 & $50,7 \pm 4,0$ & & \\
\hline $\begin{array}{l}\text { Sobrevida específica en pacientes con } \\
\text { metástasis ganglionar }\end{array}$ & & & \\
\hline Melanoma de espesor intermedio & $62,1 \pm 4,8$ & $41,5 \pm 5,6$ & $0,56(0,37 \mathrm{a} 0,84)$ \\
\hline Melanoma de espesor grueso & $48,0 \pm 7,0$ & $45,8 \pm 7,8$ & $0,92(0,53 \mathrm{a} 1,60)$ \\
\hline
\end{tabular}

HR: hazard ratio. IC95\%: intervalo de confianza del 95\%. EE: error estándar.

La frecuencia global de metástasis ganglionares en el estudio fue del $20,8 \%$, con un porcentaje similar en ambos grupos. La técnica de biopsia de ganglio centinela identificó los mismos en un $99,4 \%$ de los casos, asegurando en un $96 \%$ de los casos el correcto diagnóstico en cuanto a afectación del territorio ganglionar de drenaje.

\section{Conclusiones}

La estrategia de biopsia del ganglio centinela mejoró la sobre- vida libre de enfermedad a diez años en todos los pacientes y prolongó la sobrevida libre de enfermedad a distancia y la sobrevida específica en los pacientes con melanomas de grosor intermedio y metástasis ganglionares.

Fuentes de financiamiento: National Cancer Institute, National Institutes of Health y Australian and New Zealand Melanoma Trials Group.

\section{Comentario}

El manejo de los ganglios regionales ha sido objeto de controversias desde que Snow, hace más de un siglo propuso la linfadenectomía completa y electiva para todos los pacientes con melanoma, exponiendo a los pacientes a complicaciones y morbilidad. Sin embargo, estudios subsiguientes mostraron algún beneficio en esta conducta. Mientras este artículo se publicaba, las Sociedades de Cirugía Oncológica y Oncología Médica de Estados Unidos1 recomendaban en sus guías el uso de la investigación regional ganglionar con técnica del ganglio centinela en los melanomas de espesor intermedio, y su consideración en los de mayor espesor. Esta publicación viene a confirmar los datos que ya se tenían. Si bien la misma realmente maravilla por la demostración de los números, ya que incluyen hasta los pacientes perdidos durante estos diez años, así como la rigurosidad en distintos pasos del protocolo es necesario destacar que el análisis no fue realizado bajo el principio de intención de tratar, por lo que los resultados deben ser interpretados con cautela.

\section{Conclusiones del comentador}

Este estudio podría confirmar la factibilidad y seguridad del método de biopsia del ganglio centinela, y su relevancia como factor pronóstico a largo plazo, al permitir considerar la práctica de la adyuvancia en caso de resultar positiva. Por otro lado aleja la posibilidad de recurrencia ganglionar cuando el estudio resulta negativo, lo cual evita la morbilidad asociada a los vaciamientos ganglionares innecesarios, sobre todo en el grupo de pacientes con melanoma de espesor intermerdio.

Maria Guadalupe Pallotta Rodriguez. [ Sección Oncología, Servicio de Clínica Médica del Hospital Italiano de Buenos Aires. maria.pallotta@hospitalitaliano.org.ar ] Pallotta Rodriguez MG. La técnica de biopsia de ganglio centinela prolonga la sobrevida libre de enfermedad en pacientes con melanoma. Evid Act Pract Ambul. 2016;19(1):12. Ene-Mar. Comentado de: Morton DL, y col. Final trial report of sentinel-node biopsy versus nodal observation in melanoma. NEJM. 2014;370(7):599-609. PMID: 24521106.

Referencia:

1. Wong SL, y col. Sentinel lymph node biopsy for melanoma: American Society of Clinical Oncology and Society of Surgical Oncology joint clinical practice guideline. J Clin Oncol. 2012 Aug 10;30(23):2912-8. doi: 10.1200/JCO.2011.40.3519. Epub 2012 Jul 9. 